\title{
Risk factors for pneumocystis jirovecii pneumonia in liver transplantation recipients
}

\author{
Eun-Ki Min, Juhan Lee, Jae Geun Lee, Hyun jeong Kim
}

Department of Surgery, Severance Hospital, Seoul, Korea

Background: Pneumocystis jirovecii pneumonia (PJP), a potentially life-threatening infection occurring in immunocompromised patients, has been rarely studied in liver transplant recipients in respect to its incidence and risk factors. The aim of this study was to evaluate risk factors for PJP after liver transplantation and to address high-risk group that can possibly benefit from prolonged prophylaxis.

Methods: This is a single center, retrospective study involving 860 patients who underwent liver transplantation at Severance Hospital between January 2009 and December 2019. The incidence, risk factors and outcome of PJP were retrospectively reviewed.

Results: Among 100 patients who did not receive trimethoprim/sulfamethoxazole (TMP/SMX), 15 patients (15\%) were diagnosed with PJP, of which $80 \%$ occurred within 3 months after transplantation. Upon prescription of TMP/SMX, 25 of 760 (3.3\%) suffered PJP. In multivariate analysis, old age ( $\geq 65)$ (hazard ratio [HR], 2.842; 95\% confidence interval [Cl],1.061-7.609; P=0.038), cytomegalovirus (CMV) viremia ( $\mathrm{HR}, 3.410 ; 95 \% \mathrm{Cl}, 1.510-7.701 ; \mathrm{P}=0.003)$, and use of everolimus $(\mathrm{HR}, 2.708 ; 95 \% \mathrm{Cl}, 1.206-6.078$; $\mathrm{P}=0.016)$ were found as risk factors of diagnosis with PJP. PJP-related mortality was as high as $32 \%(8 / 25)$ in this subgroup. Conclusions: Late onset PJP occurs even after 6 to 12 months of TMP/SMX prophylaxis. This study addresses that old age, CMV viremia and use of everolimus may be risk factors for late onset PJP in liver transplant recipients. Extended duration of prophylaxis targeting high-risk recipients may be a more cost-effective strategy.

Corresponding author: Jae Geun Lee

E-mail:drjg1@yuhs.ac

(c) The Korean Society for Transplantation

This is an Open Access article distributed under the terms of the Creative Commons Attribution Non-Commercial License (http://creativecommons.org/licenses/by-nc/4.0/) which permits unrestricted non-commercial use, distribution, and reproduction in any medium, provided the original work is properly cited. 